View Article Online / Journal Homepage / Table of Contents for this issue

STENHOUSE ANL GROVES'S CONTRIBUTIONS, ETC.

L.-Contributions to the History of the Naphthalene Series. No. II. $\beta$-Naphthaquinone.

By John Stenhouse, LL.D., F.R.S., and Charles E. Groves.

\title{
B-Napthaquinone.
}

IN a paper communicated to the Chemical Society last year (1877, ii, 47), we mentioned that nitroso- $\beta$-naphthol, when converted into the corresponding amido-naphthol, and then submitted to oxidation, yielded a crystalline compound of the nature of a quinone; this we designated $\beta$-naphthaquinone, in order to distinguish it from the a-naphthaquinone previously described by one of us. Further experience has shown us that in preparing this quinone it is more advantageous to employ a considerably larger quantity of potassium dichromate than that formerly used. The proportions which we recommend are 6 parts of a saturated aqueous solution of sulphurous acid, 6 of dilute sulphuric acid ( 1 vol. acid to 2 of water), and 3 of potassium dichromate to every 2 parts of the pure nitroso- $\beta$-naphthol, the reduction and subsequent oxidation being carried out in the manner previously described (loc. cit., 52). In this way the nitrosonaphthol yields from 62-65 per cent. of its weight of the pure quinone, the theoretical quantity being 91.9. We have not ascertained the cause of this deficiency, but as the sulphur precipitated during the reduction of the nitroso- to the amido-compounds contains but very little organic matter, it is probable that the loss is occasioned chiefly by the solution of a portion of the amido- $\beta$-naphthol in the dilute solution of ammonium sulphide. This solution, however, after it has been acidified with sulphuric acid, and the hydrogen sulphide removed by heating, does not yield any quinone when mixed with a solution of potassium dichromate, as it should do if there were any quantity of the amido-naphthol present. 


\section{Nitro- $\beta$-naphthaquinone.}

It was noticed that $\beta$-naphthaquinone dissolved readily in hot dilute nitric acid, and the solution on cooling deposited a crystalline substance of a magnificent crimson colour. Since our last communication we have prepared this substance in a state of purity. The pure $\beta$-quinone (10 parts) was added to nitric acid of density 1.2 (60 parts by measure), and after the mixture had been agitated until it formed a homogeneous paste, the flask was plunged into boiling water. The quinone dissolved, and in the course of a few minutes a mass of red crystals of the new substance separated, a small quantity of nitrous fumes being at the same time evolved; this was no doubt due to secondary action. When the whole of the quinone was converted into the nitro-derivative, which only required a few minutes, the flask was removed from the water-bath and plunged into cold water, so as to stop the oxidising action of the hot nitric acid on the nitroquinone. After allowing it to stand for a couple of hours, the product was collected and washed, first with a little dilute nitric acid (density 1·2), and then with water. The crude product amounted to $70-75$ per cent. of the naphthaquinone originally taken; it was purified by dissolving it in 15 times it weight of boiling benzene, and allowing the solution to stand for 18 to 24 hours, as it separated but slowly. The hard crimson nodules which formed on the sides of the flask were finally crystallised once or twice from twice their weight of boiling glacial acetic acid, which on cooling deposited the nitro- $\beta$ naphthaquinone in magnilicent crimson plates, bearing a striking resemblance in point of colour and general aspect to chromic anhydride.

It gave the following results on analysis:-

I. '301 gram of substance gave '654 gram earbonic anhydride and .075 gram water.

II. $\cdot 197$ gram of substance gave $\cdot 429$ gram carbonic anhydride and 046 gram water.

III. 427 gram of substance gave $25.4 \mathrm{cub}$. cent. of nitrogen at $18.5^{\circ} \mathrm{c}$, and under a barometric pressure of $764.8 \mathrm{~mm}$. (corr. at $0^{\circ}$ ) equivalent to $23.443 \mathrm{cub}$. cent. dry nitrogen at $0^{\circ}$, or $\cdot 029408 \mathrm{gram}$ of nitrogen.

IV. 364 gram of substance gave $21.5 \mathrm{cub}$. cent. of nitrogen at $17 \cdot 5^{\circ}$, and under a barometric pressure of $745.9^{\circ} \mathrm{mm}$. (corr. at $0^{\circ}$ ) equivalent to $19 \cdot 830 \mathrm{cub}$. sent. dry uitrogen at $0^{\circ}$, or $\cdot 024877$ gram of nitrngen :- 
TO THE HISTORY OF THE NAPHTHALENE SERIES.

\begin{tabular}{|c|c|c|c|c|c|c|c|c|}
\hline$C_{10}$ & $=$ & 120 & $59 \cdot 11$ & $\begin{array}{c}\text { I. } \\
59 \cdot 26\end{array}$ & $\begin{array}{c}\text { II. } \\
59 \cdot 39\end{array}$ & III. & IV. & $\begin{array}{l}\text { Mean. } \\
59 \cdot 32\end{array}$ \\
\hline $\mathrm{H}_{5}$ & $=$ & 5 & $2 \cdot 46$ & $2 \cdot 77$ & $2 \cdot 59$ & - & - & $2 \cdot 68$ \\
\hline $\mathrm{N}$ & $=$ & 14 & 6.90 & - & - & 6.89 & $6 \cdot 83$ & $6 \cdot 86$ \\
\hline $\mathrm{O}_{4}$ & $=$ & 64 & $31 \cdot 53$ & - & - & - & - & - \\
\hline & & 03 & 0.00 & & & & & \\
\hline
\end{tabular}

These numbers correspond with the formula for mononitro- $\beta$. naphthaquinone, $\mathrm{C}_{10} \mathrm{H}_{5}\left(\mathrm{NO}_{2}\right) \mathrm{O}_{2}$. It has been already shown (loc. cit.) that this substance yields phthalic acid on oxidation, so that both the $\mathrm{NO}_{2}$ group and the two oxygens of the quinone must be in one and the same benzene ring (adopting Graebe's graphic formula for naphthalene), but nothing is known of their relative positions. It is not unlikely, however, if the $\beta$-quinone should really prove to be a meta-derivative, that the nitro-quinone will be found to be $\mathrm{O}: \mathrm{O}: \mathrm{NO}_{2}$ $=1: 3: 4$; but at present so little is known of the order in which the hydrogen-atoms are displaced in naphthalene, that this must be regarded as purely hypothetical.

Nitro- $\beta$-naphthaquinone is insoluble in light petroleum, almost insoluble in carbon bisulphide, slightly soluble in ether, more so in benzene and in boiling alcohol, and very readily soluble in hot glacial acetic acid; boiling with alcohol, however, appears to decompose it, as the substance does not crystallise out again as the solution cools, and on evaporation it merely deposits yellow oily drops. It dissolves pretty freely also in boiling acetic acid of 30 per cent. (density 1.04), and to a less extent in water; the orange-coloured solutions become opalescent on cooling, but do not yield crystals. It is soluble in concentrated sulphuric acid in the cold, but is at the same time decomposed, the dark-coloured liquid giving a brown precipitate when diluted with water. The nitroquinone melts at $158^{\circ}$, and when strongly heated on platinum foil burns with feeble deflagration. It undergoes reduction when treated with hydriodic acid and phosphorus, with formation of two compounds, probably the corresponding nitrohydroquinone and the amidohydroquinone, crystallising in large crimson plates and in red-brown needles; these new substances have not as yet been further examined. Aqueous solution of sulphurous acid also reduces the nitroquinone.

\section{Dinaphthyldiquinhydrone.}

It had been noticed in the preparation of $\beta$-naphthaquinone that if the freshly precipitated quinone was allowed to stand for any length of time in contact with the acid solution, it lost its bright orange colour, and became darker; this at the time was attributed to oxi- 
dation by the excess of potassic dichromate employed for its precipitation, but we have since ascertained that it is due to another cause. When the quinone is heated with mineral acids, or even when it is left in contact with them in the cold for a sufficient length of time, it is converted into a compound of a purplish-black colour analogous to quinhydrone : it is to the formation of this substance by the action of the free sulphuric acid, that the darkening of the naphthaquinone just mentioned is due, and not to oxidation.

The most convenient agent for the transformation of the $\beta$-naphthaquinone was found to be dilute sulphuric acid ( 1 vol. of acid to 2 of water). When the pure quinone (1 part) was intimately mixed with the acid (10 parts by measure), so as not to leave any portion unwetted, and allowed to stand for 24 hours, it was completely converted into the black substance, or the change could be more rapidly and conveniently effected by heating the mixture in a water-bath at about $55^{\circ}$; the quinone rapidly became dark-coloured, and at the end of ten minutes the reaction was complete. If the temperature was allowed to rise much above $60^{\circ}$ soft pasty masses were formed inclosing some of the unaltered $\beta$-quinone, so that it became somewhat difficult to convert the quinone entirely into the new compound. About twice its bulk of water was added to the mixture when cold, and the product collected and thoroughly washed with cold water to free it from adhering sulphuric acid.

The diquinhydrone thus prepared forms an indigo-black powder when dry, which is insoluble in water, carbon bisulphide, and light petroleum, almost insoluble in benzene, but soluble in glacial acetic acid; the reddish-brown solution is immediately decolorised on addition of sulphurous acid solution. The diquinhydrone dissolves in concentrated sulphuric acid with production of a deep green colour; the addition of water to this solution produces a precipitate.

Treated with reducing agents, such as hydriodic acid and phosphorus, or an aqueous solution of sulphurous acid, the diquinhydrone is converted into a colourless substance crystallising in needles, whilst with oxidising agents, as nitric acid or potassium dichromate and sulphuric acid, it yields orange-coloured prisms, so that these three substances stand in the same relation to one another as do green quinhydrone, quinol or hydroquinone, and quinone, in the benzene series.

\section{Dinaphthyldiquinone.}

The quinhydrone just described is easily converted into dinaphthyldiquinone by boiling it with nitric acid, or with a solution of potassium dichromate and sulphuric acid, but it is more conveniently prepared by pouring a solution of pure dinaphthyldiquinol in boiling 30 per cent. 
acetic acid, into nitric acid (density $1 \cdot 45$ ) in quantity sufficient to oxidise the hydroquinone. In this way the diquinone is precipitated in small lustrous prisms of a brilliant orange colour. If, however, the nitric acid be added to the acetic acid solution, instead of pouring the latter on to the nitric acid, the precipitate is of a dingy colour, probably owing to the presence of a small quantity of the diquinhydrone.

The following results were obtained on analysis of this substance dried at $100^{\circ}$ :-

I. $\cdot 196$ gram substance gave $\cdot 549$ gram carbonic anhydride and .061 gram water.

II. $\cdot 104$ gram substance gave $\cdot 2915$ gram carbonic anhydride and 033 gram water.

III. 261 gram substance gave $\cdot 732$ gram carbonic anhydride and 082 gram water.

\begin{tabular}{|c|c|c|c|c|c|c|c|}
\hline $\mathrm{C}_{10}$ & $=$ & 120 & $76 \cdot 43$ & $\begin{array}{c}\text { I. } \\
76 \cdot 39\end{array}$ & $\begin{array}{l}\text { II. } \\
76 \cdot 44\end{array}$ & $\begin{array}{l}\text { III. } \\
76 \cdot 49\end{array}$ & $\begin{array}{l}\text { Mean. } \\
76.44\end{array}$ \\
\hline $\mathrm{H}_{5}$ & $=$ & 5 & $3 \cdot 18$ & $3 \cdot 46$ & 3.52 & $3 \cdot 49$ & $3 \cdot 49$ \\
\hline $\mathrm{O}_{2}$ & $=$ & 32 & $20 \cdot 39$ & - & - & - & - \\
\hline & & 157 & $0 \cdot 00$ & & & & \\
\hline
\end{tabular}

The analytical numbers agree with the empirical formula, $\mathrm{C}_{10} \mathrm{H}_{5} \mathrm{O}_{2}$; but from a consideration of the mode of formation this should be doubled, making the formula of the dinaphthyldiquinone $\mathrm{C}_{20} \mathrm{H}_{10} \mathrm{O}_{4}$.

Dinaphthyldiquinone is insoluble in water, and but very slightly soluble, or quite insoluble, in all ordinary solvents; even boiling glacial acetic acid dissolves it but very sparingly. The diquinone is a very stable body, dissolving in boiling concentrated nitric acid, and crystallising out unchanged on cooling; by long continued boiling with the acid, however, it appears to be decomposed, and it is not improbable that a study of the products of oxidation formed in this way will yield interesting results. It is also easily soluble in warm concentrated sulphuric acid, and is precipitated again on dilution. Reducing agents convert it into the corresponding hydroquinone.

\section{Dinaphthyldiquinol.}

This compound is formed by the reduction of the diquinone or diquinhydrone, as when they are boiled with phosphorus and a solution of hydriodic acid in acetic acid of 30 per cent., or treated with aqueous solution of sulphurous acid; the quinone, however, appears to be more easily reduced than the quinhydrone. The diquinone, when allowed to stand for some time in contact with sulphurous acid solution, becomes converted into a mass of colourless crystals of the 
diquinol; the quinhydrone does not appear to be produced as an intermediate stage in this reaction, for at no time could the black crystals of this compound be distinguished in the mixture. A very convenient method of preparation is to convert the $\beta$-naphthaquinone into the diquinhydrone by treatment with dilute sulphuric acid in the manner previnusly described, and when the mixture is cold, to add about twice its bulk of a saturated aqueous solntion of sulphurous acid, and allow the whole to stand for 24 hours, when the black diquinhydrone will be found to be entirely converted into a mass of snow-white needles of dinaphthyldiquinol, provided the $\beta$-naphthaquinone originally employed was quite pure: these merely require to be thoroughly washed to remove sulphuric acid, and crystallised once from dilute acetic acid, to which a few drops of sulpharous acid solution have been added, to be quite pure. If impure $\beta$-quinone has been used for the preparation of the diquinhydrone, the diquinol will be coloured and somewhat difficult to purify; repeated conversion into the diquinone and reconversion into the diquinol will, however, usually suffice to render it c lourless.

Dinaphthyldiquinol, $\mathrm{C}_{20} \mathrm{H}_{14} \mathrm{O}_{4}$, or $\mathrm{C}_{20} \mathrm{H}_{10}(\mathrm{OH})_{4}$, forms colourless needles, which, on drying, become darkened from partial oxidation. It melts at $176-178^{\circ}$, is almost insoluble in water, moderately soluble in 30 per cent. acetic acid, easily in the glacial acid. It is only slightly soluble in benzene, bisulphide of carbon, and ether. The analytical results were as follows:-I. $\cdot 244$ gram substance, gave 676 gram carbonic anhydride and 0905 gram water.

$$
\begin{array}{lrr}
\mathrm{C}_{20}=240 & 75 \cdot 47 & 75 \cdot 56 \\
\mathrm{H}_{14}=14 & 4 \cdot 40 & 4 \cdot 12 \\
\mathrm{O}_{4}=\frac{64}{318} & \frac{20 \cdot 13}{100 \cdot 00} & -
\end{array}
$$

These numbers show a deficiency in hydrogen for the formula, $\mathrm{C}_{20} \mathrm{H}_{14} \mathrm{O}_{4}$, which is what might be expected from the comparative ease with which the substance becornes oxidised.

\section{Mode of Formation of Dinaphthyldiquinhydrone.}

The conversion of $\beta$-naphthaquinone into the black diquinhydrone, under the influence of acids, would seem to take place by the condensation of 2 molecules of the quinone, with elimination of two atoms of hydrogen which unite with two out of the four oxygen atoms in the double quinone-molecule to form a quinbydrone-

$$
\mathrm{C}_{10} \mathrm{H}_{6} \mathrm{O}_{2}+\mathrm{C}_{10} \mathrm{H}_{6} \mathrm{O}_{2}=\mathrm{C}_{20} \mathrm{H}_{10} \mathrm{O}_{2}(\mathrm{OH})_{2} \text {, }
$$


or regarding the quinhydrone as formed by the union of a molecule of the quinone with one of the quinol:

$$
2 \mathrm{C}_{10} \mathrm{H}_{6} \mathrm{O}_{2}+2 \mathrm{C}_{10} \mathrm{H}_{6} \mathrm{O}_{2}=\mathrm{C}_{20} \mathrm{H}_{10} \mathrm{O}_{4} \cdot \mathrm{C}_{20} \mathrm{H}_{10}(\mathrm{OH})_{4} \text {. }
$$

This view is strongly supported by the fact that $\beta$-naphthaquinone yields its own weight of the diquinhydrone. Two experiments were made, in each of which 500 gram of pure $\beta$-naphthaquinone was converted into the diquinhydrone by treatment with dilute sulphuric acid: the products collected, washed thoroughly, and dried, weighed $\cdot 498$ and $\cdot 495$ gram respectively.

It was evident that the results of an analysis of the diquinhydrone for hydrogen (taking into consideration its high molecnlar weight, and that in all probability it readily alters by oxidation) would not yield satisfactory evidence as to whether this is a correct explanation of the change which takes place. It seemed probable, however, that if the hydrogen-atoms external to the nucleus (that is, those not directly attached to carbon-atoms) could be determined, it would at once show whether the reaction was of the nature above indicated: a means of doing this is afforded by the action of bromine in aqueous solution, which readily oxidises the diquinhydrone, but does not attack the diquinone. A weighed quantity of the pure $\beta$-naphthaquinone was accordingly converted into the black diquinhydrone, and agitated with excess of bromine-water of known strength for a few minutes, whereby it was converted into the diquinone; the excess of bromine was then estimated by potassic iodide and hyposulphate solution in the usual manner. In this way it was found in two experiments that 200 gram of pure $\beta$-naphthaquinone, after conversion into the diquinhydrone, required $\cdot 100$ and $\cdot 098$ of bromine respectively to oxidise it to the new quinone; the theoretical amount for

$$
\mathrm{C}_{20} \mathrm{H}_{10} \mathrm{O}_{2}(\mathrm{OH})_{2}+\mathrm{Br}_{2}=\mathrm{C}_{20} \mathrm{H}_{10} \mathrm{O}_{4}+2 \mathrm{HBr}
$$

being 101 gram bromine. There can be little doubt, therefore, that the black substance is a diquinhydrone analogous to the green quin. hydrone of the benzene series.

Although it was almost certain-the nature of the diquinone and diquinhydrone being established-that the formula of the diquinol was $\mathrm{C}_{20} \mathrm{H}_{10}(\mathrm{OH})_{4}$, notwithstanding the analytical results showed a deficiency in the hydrogen; yet it was thought advisable to make experiments by oxidation with bromine-water in a manner similar to those detailed for the diquinhydrone, with a view to ascertain whether it actually contained four $\mathrm{OH}$-groups. From the readiness with which the substance oxidises, however, the results were not very accordant. A weighed quantity of the dinaphthyldiquinone, $\mathrm{C}_{20} \mathrm{H}_{10} \mathrm{O}_{4}$, was reduced by sulphurous acid solution, washed thoroughly, collected on a vacuum 
filter, and the product, which had already become slightly coloured, was dissolved in hot glacial acetic acid; the rather dark-coloured solution was then poured into an excess of bromine-water, and the excess of free bromine determined as before. Three experiments gave $98: 88.5$ and 85 of bromine per 100 of the diquinone, whilst theory requires 101.9. The diquinone formed in these oxidation experiments was examined, to ascertain if any bromine substitution-derivative had been formed, but it was found to be free from bromine.

The compound, $\mathrm{C}_{20} \mathrm{H}_{10}(\mathrm{OH})_{4}$, may be regarded as a tetrahydric alcohol of the hydroquinone or quinol species, derived from a dinaphthyl, $\mathrm{C}_{10} \mathrm{H}_{7} . \mathrm{C}_{10} \mathrm{H}_{7}$, by the displacement of four hydrogen-atoms by four OH-groups, and is a member of a new class of bodies, the diquinols. We have, therefore, called it dinaphthyldiquinol, and the corresponding quinone-compound, dinaphthyldiquinone.

We have ascertained that benzoquinone and $\alpha$-naphthaquinone also yield diquinones and diquinols by condensation, and have no doubt that other quinones will yield corresponding compounds. We are at present engaged in studying this interesting group.

Our thanks are due to Mr. F. Sear for the care with which he has done much of the analytical work in connection with this paper. 